\title{
O COMÉRCIO DE FRUTOS DE TUCUMÃ (Astrocaryum aculeatum G. Mey - ARECACEAE) NAS FEIRAS DE MANAUS (AMAZONAS, BRASIL) ${ }^{1}$
}

\author{
ADRIANO AMIR DIDONET ${ }^{2}$, ISOLDE DOROTHEA KOSSMANN FERRAZ ${ }^{3}$
}

RESUMO - Tucumã é uma palmeira amazônica. A polpa dos frutos é comestível sem cozimento e muito apreciada como recheio de sanduíches na culinária regional, possui alto valor nutritivo e destaque no teor de vitamina A. O presente estudo teve como objetivo avaliar aspectos da comercialização dos frutos e da polpa de tucumã em feiras e mercados de Manaus. Durante o estudo de um ano, foram levantadas as procedências e preços dos frutos e estimada a quantidade comercializada em seis feiras e mercados de Manaus. Os dados de procedência, quantidade, preço e outras informações relacionadas foram obtidos através da aplicação mensal de um questionário semiestruturado aos comerciantes. O preço real e sua evolução histórica, desde 1995, foram deduzidos por comparação com estudos anteriores. Entre maio de 2011 e abril de 2012, os frutos foram provenientes de 20 diferentes localidades a uma distância de até mil km de Manaus. Os locais mais citados foram os municípios de Itacoatiara, Terra Santa, Rio Preto da Eva, Autazes e a bacia do Rio Madeira. A alternância das procedências garantiu o abastecimento contínuo ao longo do ano. Entretanto, uma redução do número de procedências e da quantidade de frutos comercializados foi verificada entre setembro e novembro. A sazonalidade da oferta foi refletida nos preços dos frutos e da polpa. Durante os 12 meses, foi comercializado um total de 367,8 toneladas de frutos, com 30,7 toneladas de média mensal. Cerca da metade $(53 \%)$ desta quantia foi descascada durante a jornada de trabalho dos feirantes e comercializada apenas a polpa. Neste estudo e baseado em informações anteriores, tucumã foi sempre um dos frutos mais caros dos mercados de Manaus. Entre 1995 e 2012, os valores reais aumentaram substancialmente, tanto da saca $(230 \%)$, com cerca de $41 \mathrm{~kg}$, como da dúzia de frutos $(316 \%)$ e do $\mathrm{kg}$ da polpa $(253 \%)$. Os resultados indicam que o mercado de tucumã continua em expansão. Aparentemente, a demanda é maior do que a oferta e, dessa forma, há necessidade de aumento da produção.

Termos para indexação: Amazônia central, produto florestal não madeireiro, palmeira; mercado.

\section{FRUIT TRADE OF TUCUMA (Astrocaryum aculeatum G. Mey - ARECACEAE) AT LOCAL MARKET-PLACES IN MANAUS (AMAZONAS, BRAZIL)}

\begin{abstract}
Tucumã is an Amazonian palm tree. The raw pulp of the fruit is edible and highly appreciated as sandwich filling in local culinary. The pulp of high nutritional value has significant vitamin A content. This study aimed to evaluate aspects of the trade of tucumã fruit and pulp at fairs and markets of Manaus. During the one-year study, the provenances and price variation of the fruits were registered and estimated the amount delivered to six markets and fairs of Manaus. Data on provenance, quantity, value, and related information were obtained by applying monthly a semi-structured questionnaire to the salesmen. The real price and its historical evolution since 1995 were deduced by comparison with previous studies. Between May 2011 and April 2012, the fruits came from 20 different provenances, within a distance of up to thousand $\mathrm{km}$ from Manaus. The most cited sources were the municipalities of Itacoatiara, Terra Santa, Rio Preto da Eva, Autazes and the Madeira River Basin. The alternation of the provenances guaranteed continuous supply throughout the year. However a lesser number of provenances and a narrow market of the fruits were stated between September and November. The seasonality of supply was reflected in the values of fruit and pulp. During the 12 months were traded a total of 367.8 tons of fruits, at a monthly average of 30.7 tons. About half $(53 \%)$ of this amount was peeled during the working hours of the salesmen and only the pulp was offered for sale. In this study and as far as information was available, tucumã was always one of the most expensive fruits of the Manaus markets. Between 1995 and 2012 actual values had increased substantially, as of the bag $(230 \%)$ with about $41 \mathrm{~kg}$, the dozen of fruits $(316 \%)$ and the pulp $\mathrm{kg}(253 \%)$. The results show that the tucumã trade continues to expand. The demand seems to be greater than the supply and an increase in fruit production is needed.
\end{abstract}

Index terms: Central Amazon, non timber forest products, palm, market.

\footnotetext{
${ }_{1}^{1}$ (Trabalho 108-13). Recebido em: 13-03-2013. Aceito para publicação em : 13-02-2014.

${ }^{2} \mathrm{MSc}$. em Ciências de Florestas Tropicais - INPA, Manaus- AM. E-mail: adriano.didonet@inpa.gov.br

${ }^{3}$ Dra. Pesquisadora do INPA/CBIO, Manaus-AM. E- mail: iferraz@inpa.gov.br
} 


\section{INTRODUÇÃO}

Existem duas espécies popularmente conhecidas como tucumã, cujos frutos são comestíveis e comercializados na região Norte do Brasil: (i) Astrocaryum vulgare Mart., que tem demanda estável centrada na região de Belém; e (ii) Astrocaryum aculeatum G. Mey., também conhecida como tucumã-açu, com demanda consolidada e crescente na Amazônia central, tendo como principal mercado a cidade de Manaus (CLEMENT et al., 2005).

O A. aculeatum, objeto deste estudo, é uma palmeira nativa das terras firmes da Amazônia que ocorre no norte da Bolívia, na Amazônia colombiana, Venezuela, Guiana, Suriname e no Brasil, em toda a Amazônia ocidental até o oeste do Pará, no Mato Grosso e Roraima (KAHN, 2008). Essa palmeira é muito frequente em Manaus e em sua periferia, onde é encontrada entre cultivos, vegetações secundárias, pastos e áreas abertas resultantes de atividade humana (KAHN; MOUSSA, 1997).

O tucumã tem sido usado desde a época dos Ameríndios até os dias de hoje pelos povos amazônicos, com aproveitamento de praticamente todas as partes da planta: (i) o estipe serve à construção civil e para confecção de arco; (ii) das folhas, além do emprego na manufatura de cestos, chapéus, abanadores e esteiras, é extraído o "tucum", fibra de alta qualidade com a qual são confeccionadas redes para dormir, sacos, bolsas e redes de pesca; (iii) com menor expressividade, é extraído sal das folhas; (iv) o meristema apical (palmito) é usado na alimentação; (v) o mesocarpo do fruto é comestível, e o endocarpo é usado no artesanato e como material combustível; (vi) a semente serve de complemento alimentar para animais domésticos, além de extrair-se dela óleo que pode ser utilizado como biodiesel e como insumo na indústria cosmética e alimentícia (KAHN; MOUSSA, 1999; COSTA et al., 2005).

Foram os frutos que tornaram o tucumã emblemático em Manaus, ao ponto de ter sido considerado a palmeira que melhor representa a capital amazonense (KAHN; MOUSSA, 1997). Em torno desse fruto, desenvolveu-se um importante mercado na região central da Amazônia (SCHROTH et al., 2004), que gerou emprego e renda para um contingente da população que vive na capital e outros municípios onde a palmeira é encontrada. Sua polpa é muito apreciada localmente e aproveitada na composição de sorvete, pastas e inúmeros outros produtos (KAHN; MOUSSA, 1999), destacando-se como recheio de tapioca e sanduíche. O "X-caboquinho", um sanduíche típico popular, é um impor- tante produto dos cafés regionais que surgiram na década de 1990 e atualmente estão presentes em feiras livres, terminais rodoviários e em diversos pontos das calçadas de Manaus. A partir daí, a polpa do tucumã tornou-se um ingrediente do "fast food" local (COSTA et al., 2005), que além da crescente importância no Estado do Amazonas, possui potencial para conquistar novos mercados fora da região (LOPES et al., 2009).

Em Manaus, o fruto é comumente comercializado sob duas formas: (i) in natura, vendido em dúzia ou cento; e (ii) beneficiado localmente pelos próprios comerciantes, sendo a polpa comercializada por peso. Por se tratar de um produto regional de origem florestal, raramente é vendido em supermercados, mas é abundante em feiras livres, mercados e pontos estratégicos de venda nas ruas de Manaus, onde pode ser encontrado durante todo o ano (COSTA et al., 2005). Essa oferta permanente deve-se ao fato de os frutos serem oriundos de diversas localidades, onde ocorrem pequenas diferenças no período de frutificação, proporcionadas pela variação na estação chuvosa (KAHN; MOUSSA, 1999). No entanto, embora permanente, a oferta de frutos ao longo do ano é heterogênea, o que determina uma variação em seu preço.

O mercado de Manaus é atendido quase exclusivamente pela exploração extrativista. A importância do extrativismo em comparação ao sistema de cultivo é favorecida pela ocorrência comum desta palmeira em áreas degradadas (SCHROTH et al., 2004) e provavelmente pela germinação lenta, devido à dormência das sementes (GENTIL, 2005) e o lento desenvolvimento da planta (FAO, 1987). Diante disso, a comercialização dessa espécie pode contribuir na redução da pobreza em comunidades marginalizadas e dependentes da floresta amazônica, contribuindo com seu bem-estar e renda (MARSHALL et al., 2003).

Embora a maioria dos trabalhos dedicados a essa palmeira cite sua importância e potencial econômicos, informações quantitativas referentes ao seu mercado regional não são disponíveis, dificultando ações públicas e privadas visando ao planejamento e desenvolvimento de sua cadeia produtiva.

Dessa forma, são apresentados neste trabalho alguns aspectos da comercialização dos frutos de tucumã in natura e da polpa em feiras livres e mercados da cidade de Manaus entre os meses de maio/2011 e abril/2011, com ênfase na sazonalidade da oferta, dos preços desse produto, bem como sua procedência. Baseado em informações disponíveis, buscou-se discutir a evolução da oferta e da demanda desse produto entre 1995 e 2012. 


\section{MATERIAL E MÉTODOS}

O estudo foi realizado entre os meses de maio de 2011 e abril de 2012 em feiras livres e mercados de Manaus, capital do Estado do Amazonas $\left(3^{\circ} \mathrm{S}\right.$, $\left.60^{\circ} \mathrm{W}\right)$. O município situa-se na confluência dos rios Negro e Solimões e possui limites com os municípios de Presidente Figueiredo, Rio Preto da Eva, Itacoatiara, Careiro da Várzea, Iranduba e Novo Airão. Seu território abrange $11.458,5 \mathrm{~km}^{2}$, dos quais 3,3\% são considerados área urbana. Segundo dados do IBGE (2010), Manaus continha 1.718.584 habitantes em 2010.

Em janeiro de 2012, estavam registrados 41 feiras livres e mercados na Secretaria Municipal de Produção e Abastecimento (SEMPAB); entretanto, foram identificados comerciantes regulares de tucumã em apenas 3 feiras livres e 3 mercados: (i) Feira Municipal Produtor da Zona Leste; (ii) Mercado Municipal Dorval Porto; (iii) Feira Municipal do Parque 10; (iv) Feira Municipal Panair; (v) Mercado Municipal Adolpho Lisboa; e (vi) Mercado Municipal Walter Rayol. Sendo assim, foi aplicado um questionário semiestruturado mensalmente, nesses seis locais, a todos os comerciantes de tucumã, o qual continha perguntas sobre: (i) o preço no atacado e no varejo, (ii) a procedência dos frutos, (iii) o ponto de abastecimento, (iv) a quantidade comercializada e (v) observações pertinentes à descrição do comércio de tucumã nesses espaços. No total, foram aplicados 120 questionários a 16 comerciantes. O estudo foi aprovado pelo Comitê de Ética em Pesquisa com seres humanos do INPA (CEP-INPA).

$\mathrm{O}$ peso de frutos comercializado mensalmente foi estimado com base no número de sacas vendidas semanalmente. $\mathrm{O}$ peso médio da saca foi determinado por amostragem aleatória. A cada mês, 3 sacas ainda fechadas foram pesadas com uma balança de $1 \mathrm{~kg}$. A quantidade de polpa de uma dúzia e de uma saca de frutos foi estimada com base na massa fresca $(0,001 \mathrm{~g})$ dos verticilos, epicarpo (casca), mesocarpo (polpa) e pirênio (caroço composto por endocarpo duro e semente). Para a biometria, foram utilizados frutos maduros obtidos entre maio de 2011 e janeiro de 2012. Para cada procedência, tomou-se uma amostra de 30 frutos em perfeito estado de maturação oriundos de 10 localidades: (i) Autazes; (ii) Barreirinha; (iii) Codajás; (iv) Itacoatiara; (v) Manaus; (vi) Rio Preto da Eva; (vii) Uucará do Amazonas; (viii) Boa Vista de Roraima; (ix) Terra Santa do Pará; e (x) Rio Madeira (Figura 1). Buscou-se amostrar os frutos aleatoriamente de sacas da mesma procedência.
Em todas as entrevistas, foi realizado o levantamento do preço dos frutos no atacado, correspondente a uma saca, e no varejo, a uma dúzia e a $1 \mathrm{~kg}$ de polpa. A evolução histórica (entre 1995 e 2012) do valor da saca, da dúzia de frutos e do $\mathrm{kg}$ da polpa foi estimada com base nos preços encontrados na literatura. Os preços em valores nominais foram convertidos em valores reais através do Índice Geral de Preços (IGP-DI), publicados na Revista Conjuntura Econômica da Fundação Getúlio Vargas, com base no ano da primeira publicação (1995 para a dúzia e 2003 para a saca e o kg da polpa). O valor agregado pela venda no varejo (dúzia de frutos) ou pela despolpa dos frutos foi avaliado com base na quantidade de polpa contida em uma saca ou uma dúzia de frutos e expresso em $\mathrm{R} \$ / \mathrm{kg}$ de polpa. Para análise gráfica da sazonalidade, utilizou-se do programa Sigmaplot 11.0 .

A importância de cada município fornecedor foi avaliada pelas indicações obtidas nas entrevistas mensais, sendo a razão entre o número de indicações de uma dada procedência pelo número total de indicações no mês ou no ano, expressa em percentagem (\%). Muitos entrevistados destacaram a região do Rio Madeira como fornecedora, sem distingui-la em municípios. Entretanto, os municípios de Nova Olinda do Norte e Manicoré, adjacentes a esse rio, também foram indicados. Para análise de importância, tais municípios foram agrupados em "Rio Madeira".

\section{RESULTADOS}

\section{Oferta}

Foram comercializadas no ano $367,8 \mathrm{t}$ de frutos de tucumã nas feiras e mercados de Manaus estudados, das quais 46,6\% (171,1 t) foram revendidos no varejo, geralmente em dúzia de frutos, e 53,4\% (196,7 t) foram beneficiados para a venda da polpa. O preço nominal da saca no atacado variou ao longo do ano entre $\mathrm{R} \$ 30,00$ e $\mathrm{R} \$ 180,00$, sendo o valor mais comum $\mathrm{R} \$ 80,00$. No varejo, a dúzia foi revendida, em média, a $\mathrm{R} \$ 3,80( \pm 1,15)$, e o kg da polpa, em média, a $\mathrm{R} \$ 31,50( \pm 6,50)$, sendo os preços mais comuns $\mathrm{R} \$ 3,00$ pela dúzia de frutos e R\$ 30,00 por um kg de polpa (Tabela 1).

Os frutos de tucumã foram oferecidos em todos os meses, entre maio de 2011 e abril de 2012. Os feirantes indicaram como procedência 17 municípios do Estado do Amazonas, um do Pará e um de Roraima; além disso, destacaram a bacia do Rio Madeira como uma importante região fornecedora para o mercado de Manaus, sem detalhamento dos municípios ao longo deste rio, 
que são no Brasil: Apuí, Manicoré, Borba, Nova Olinda do Norte e Novo Aripuanã no Amazonas e Porto Velho em Rondônia (Figura 1).

O período de abastecimento ao longo do ano variou consideravelmente entre as procedências. Enquanto o município de Terra Santa forneceu frutos durante 10 meses ao mercado de Manaus, outros como Coari, Fonte Boa, Manaus, Nhamundá, Silves, Tefé e Urucurituba foram indicados somente durante um dos meses deste estudo (Tabela 2). Ao longo do ano, foram mencionados entre 6 e 10 procedências de frutos em cada mês, considerando à parte o mês de outubro, caracterizado pela interrupção do fornecimento pela grande maioria das fontes (Figura 2). A mudança mensal de posição no ranking de procedências indica uma rotatividade, importante para a continuidade do abastecimento ao longo do ano (Tabela 2). Essa rotatividade, provavelmente foi, provocada por um ou mais fatores limitantes relacionados não só à produção como também à infraestrutura disponível para garantir a distribuição do tucumã em escala regional.

As localidades com maior importância para o abastecimento de Manaus foram Itacoatiara e Autazes, seguidas por Terra Santa, Rio Madeira e Rio Preto da Eva. Juntas, representaram nas entrevistas $59 \%$ das indicações de procedência dos frutos (Tabela 2). Itacoatiara, Rio Preto da Eva e Autazes destacaram-se no período entre dezembro e julho, enquanto Terra Santa e Rio Madeira foram mais importantes no período entre agosto e novembro.

A região do Rio Madeira e Terra Santa destacou-se como fornecedora durante o período de escassez, entre setembro e novembro (Figura 2). Juntas, representaram em setembro $64 \%$, em outubro $100 \%$ e em novembro $50 \%$ das procedências indicadas nas entrevistas. Autazes, embora faça parte da Bacia do Rio Madeira, apresentou comportamento de abastecimento diferenciado dos demais municípios da bacia (Tabela 2), provavelmente devido à proximidade com Manaus e à facilidade de acesso através das rodovias BR319 e AM-254. É importante ressaltar que a região geográfica onde está situada a bacia do Rio Madeira apresenta um índice pluviométrico anual de 2.500$2.750 \mathrm{~mm}$, superior à média da Amazônia Central de 2.250-2.500 mm/ano (NIMER, 1972). Como a fenologia do tucumã está relacionada à variação pluviométrica, frutificando no período chuvoso (SCHROTH et al., 2004), a particularidade climática da região do Rio Madeira pode estar contribuindo para sua diferenciação sazonal em relação às outras procedências. Relacionada à pluviosidade elevada, foi constatada alta diversidade local, especialmente de palmeiras (Arecaceae), na região do Rio Madeira.
Assim, esta bacia foi definida como área prioritária para conservação e coleta de material destinado ao melhoramento genético de espécies dessa família (LLERAS et al., 1983).

A maioria dos municípios fornecedores estava localizada a uma distância de até $550 \mathrm{~km}$ de Manaus, na região do médio Rio Amazonas até o oeste do Pará (Figura 1). No entanto, os feirantes receberam também frutos de Jutaí a cerca de 1.000 $\mathrm{km}$ de Manaus. A origem dos frutos diverge de um estudo realizado entre 1995 e 1997 (KAHN; MOUSSA, 1999); neste, foi indicado o próprio município de Manaus como fornecedor durante a safra (fevereiro a agosto), enquanto somente na entressafra (setembro a janeiro) os frutos chegaram de municípios adjacentes a Manaus. No presente trabalho, verificou-se que os frutos de Manaus foram comercializados apenas em janeiro e tiveram pouca importância para os feirantes, inferior até aos procedentes de Jutaí (Tabela 2). Esta informação indica um potencial de abastecimento interno subutilizado ou ainda uma produção insipiente, incapaz de abastecer o mercado atual. A situação de 2011/2012 revelou também que, além da distância, outros aspectos como as relações entre fornecedores e feirantes devem ser levados em consideração ao se analisar os entraves comerciais. Como exemplo, foi observado que os fornecedores de Jutaí tiveram sua participação garantida no mercado de Manaus devido ao crédito dado aos comerciantes que não possuíam capital de giro suficiente para a compra de frutos à vista.

\section{Sazonalidade}

Ainda que Manaus tenha sido abastecida com frutos de tucumã durante todo o ano, foi observada uma sazonalidade em relação à procedência, quantidade de frutos comercializados e aos preços dos produtos. Em 9 meses do ano (dezembro a agosto), foram indicados pelos comerciantes entre 7 e 10 procedências, abastecendo o mercado a cada mês. Os municípios mais importantes nesse período foram Itacoatiara, Rio Preto da Eva, Autazes, Barreirinha e Careiro Castanho. A quantidade de frutos que entrou mensalmente no mercado de Manaus se manteve estável em torno de 34,5 $( \pm 4,6)$ $\mathrm{t}$, com destaque para agosto $(42,8 \mathrm{t})$, mês com maior quantidade comercializada (Figura 2). Nesse período, o preço da saca variou $26 \%$ em torno da média de $\mathrm{R} \$ 72,48( \pm 16,77)$, principalmente devido a um aumento no mês de junho (R $\$ 108,33)$. Entretanto, os feirantes mantiveram os preços da revenda dos frutos e do $\mathrm{kg}$ da polpa mais estáveis, com variação dos preços no varejo de apenas $4 \%$ e $6 \%$ em torno das médias de $\mathrm{R} \$ 3,55( \pm 0,15)$ e R\$ 28,84 ( $\pm 1,69)$, respectivamente (Tabela 1). Entre setembro e novembro, especialmente em 
outubro, observou-se uma redução no número de procedências e na quantidade de tucumã comercializada (Figura 2; Tabela 2) e paralelamente um aumento do preço dos produtos (Figura 3). Em outubro $42 \%$ dos entrevistados deixaram de comercializar tucumã. Uma redução no número de comerciantes de tucumã nos mercados e feiras, no segundo semestre do ano, também foi mencionada em um estudo recente (RABELO, 2012). Vale ressaltar a afirmação de um entrevistado que relacionou o desinteresse de comercializar frutos de tucumã nessa época à baixa qualidade dos frutos oferecidos (referindo-se aos frutos provenientes do Rio Madeira) e não à falta de frutos ou ao aumento dos preços.

Corroborando a lei da oferta e da procura, observaram-se em outubro os preços nominais mais altos, tanto no atacado como no varejo: entre agosto e outubro, meses com o máximo e o mínimo ofertado, houve aumento do preço da saca (109\%), dúzia do fruto (91\%) e polpa (42\%) (Figura 3). Além da redução da oferta, o período de águas baixas em setembro e outubro no Rio Madeira (CARDOSO; FREITAS, 2007), no qual a navegabilidade é dificultada, pode ter contribuído com o aumento do preço e talvez com a redução da qualidade dos frutos devido ao tempo de transporte.

\section{Histórico do preço}

Já no final da década de 1980, o fruto de tucumã era um dos produtos mais caros do mercado de Manaus (FAO, 1987). Cerca de dez anos depois, Kahn e Moussa (1999) afirmaram ser um artigo de luxo e relataram o valor de $\mathrm{R} \$ 5,00$ por dúzia, superior a $1 \mathrm{~kg}$ de maçã importada da região $\mathrm{Sul}$ do Brasil. Comparando a evolução dos preços, verificou-se que, desde 1995 (KAHN; MOUSSA, 1999), houve um aumento de $316 \%$ no valor real ( $\mathrm{R} \$ 15,78)$ da dúzia. Com relação ao valor real da saca $(\mathrm{R} \$ 133,32)$ e do $\mathrm{kg}$ da polpa ( $\mathrm{R} \$ 50,60)$, houve um aumento de $230 \%$ e $253 \%$, respectivamente, entre 2003 e 2011 (Tabela 3).

Neste estudo, um quilograma da polpa foi quatro vezes maior do que um quilograma do tambaqui (Colossoma macropomum Cuvier), um dos peixes mais apreciados localmente. Mesmo diante dos elevados preços, o tucumã foi muito procurado e facilmente escoado, o que indica uma demanda superior à oferta.

Uma avaliação do mercado do açaí (Euterpe oleraceae Mart.) em Belém, que apresenta relações econômico-culturais semelhantes ao tucumã em Manaus, demonstrou uma correlação muito próxima entre a quantidade comercializada de frutos e o aumento da população urbana (BRONDÍZIO et al., 2002). Semelhantemente, pode-se considerar que o crescimento de $22 \%$ da população urbana de Manaus entre 2000 e 2010 (IBGE, 2010), provavelmente, causou um aumento da demanda por produtos regionais, em especial por tucumã.

$\mathrm{O}$ extrativismo, questionado quanto a sua capacidade de atender a grandes mercados (HOMMA, 1982), continua sendo o principal sistema de produção dessa fruta (LOPES et al., 2009). A restrição na oferta, determinada pelo estoque natural, apresenta-se como o principal obstáculo ao desenvolvimento do mercado da maioria dos produtos florestais não madeireiros no Brasil (HOMMA, 1982; ALMEIDA et al., 2009). De acordo com a teoria do extrativismo na Amazônia (HOMMA, 1982), o mercado do tucumã pode estar situado na fase na qual a oferta é restrita ao estoque natural, e os preços devem continuar a elevar-se, dada a incapacidade do setor em atender ao crescimento da demanda. Técnicas de manejo adequadas das populações naturais e a domesticação in situ de tucumã podem ser estratégias com baixo risco para aumentar a capacidade de suporte e melhoria da qualidade dos frutos (SCHROTH et al., 2004), a exemplo do manejo dos bacurizais no nordeste paraense (HOMMA, 2007). Considerando a abrangência geográfica do tucumã (Figura 1), outras regiões podem ser identificadas para a expansão da produção a curto e médio prazos, entretanto devem ser sanados alguns problemas vinculados à comercialização de produtos do extrativismo na Amazônia, como a deficiência na organização da comunidade, no gerenciamento da produção e comercialização, no manejo e beneficiamento dos produtos e na carência de comunicação, e infraestrutura de transporte, a qual dificulta e eleva o custo ao deslocar o produto ao mercado (BELCHER; SCHRECKENBERG, 2007). Por fim, em longo prazo, o alto preço da polpa do tucumã, provavelmente, induzirá plantios racionais (HOMMA, 1982), especialmente na proximidade de Manaus e outros centros urbanos onde há concentração de áreas degradadas e maiores demandas. Nesses locais, a ocorrência de populações naturais adensadas de tucumanzeiro indica a viabilidade de seu cultivo. Dessa forma, recomenda-se o manejo adequado dessa espécie em sistemas agroflorestais de forma a garantir o abastecimento do mercado, a conservação da floresta e do modo de vida amazônico. 


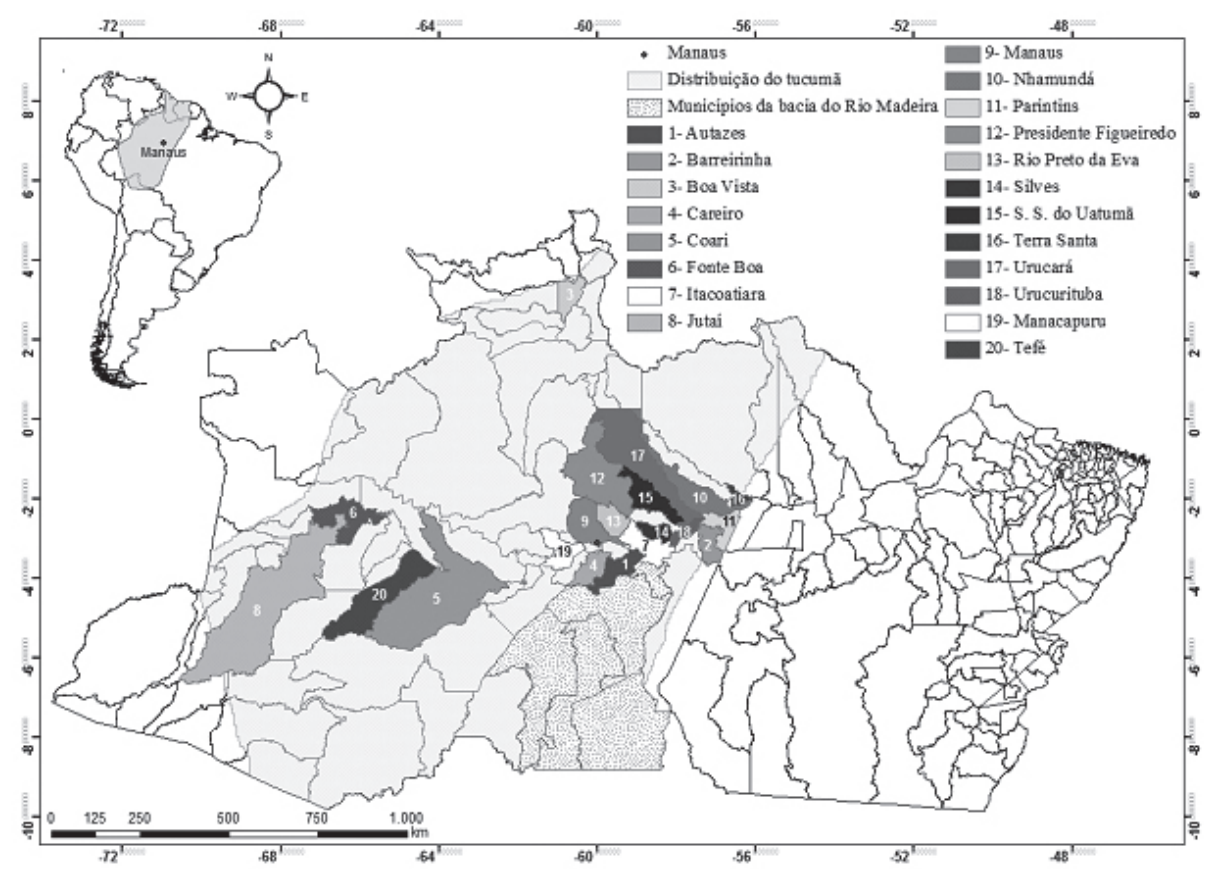

FIGURA 1 - Distribuição geográfica de Astrocaryum aculeatum (modificado de HENDERSON et al., 1995) e as localidades que forneceram frutos às feiras e mercados de Manaus-AM, entre maio/2011 e abril 2012.

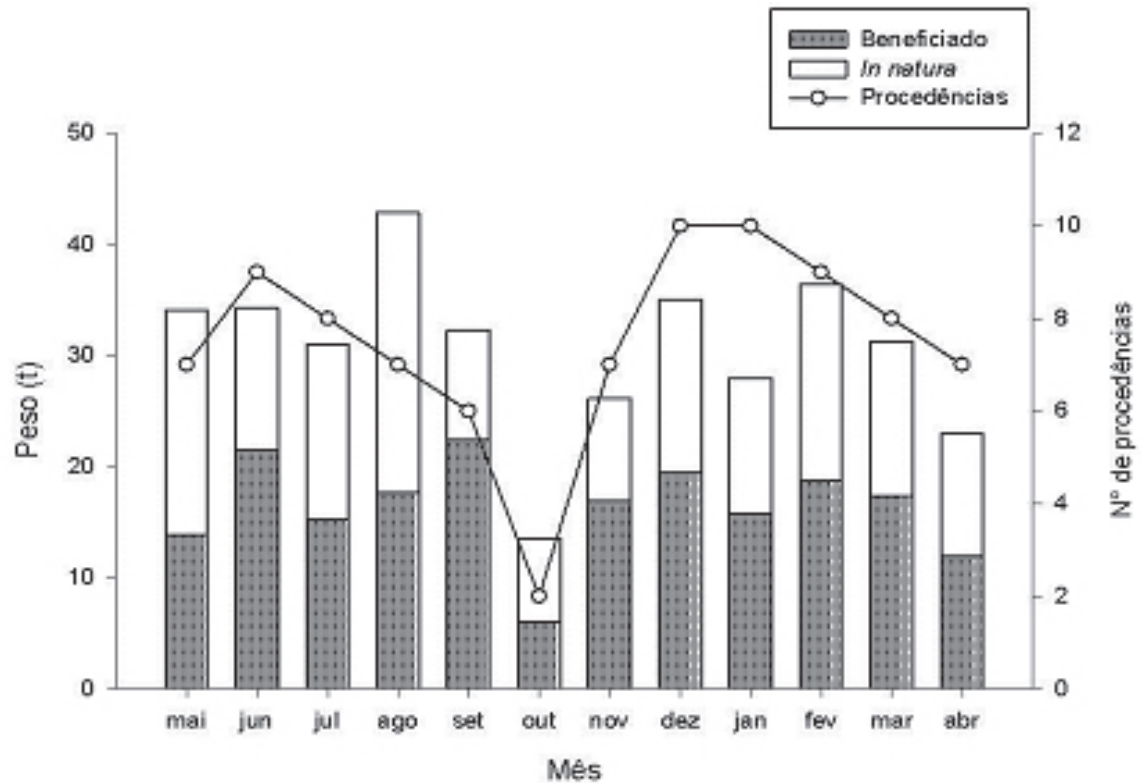

FIGURA 2 - Número de procedências (linha) e peso (coluna) de frutos de Astrocaryum aculeatum, comercializado in natura (saca ou dúzia) ou beneficiado (polpa), em feiras e mercados de Manaus-AM, entre maio/2011 e abril/2012. 


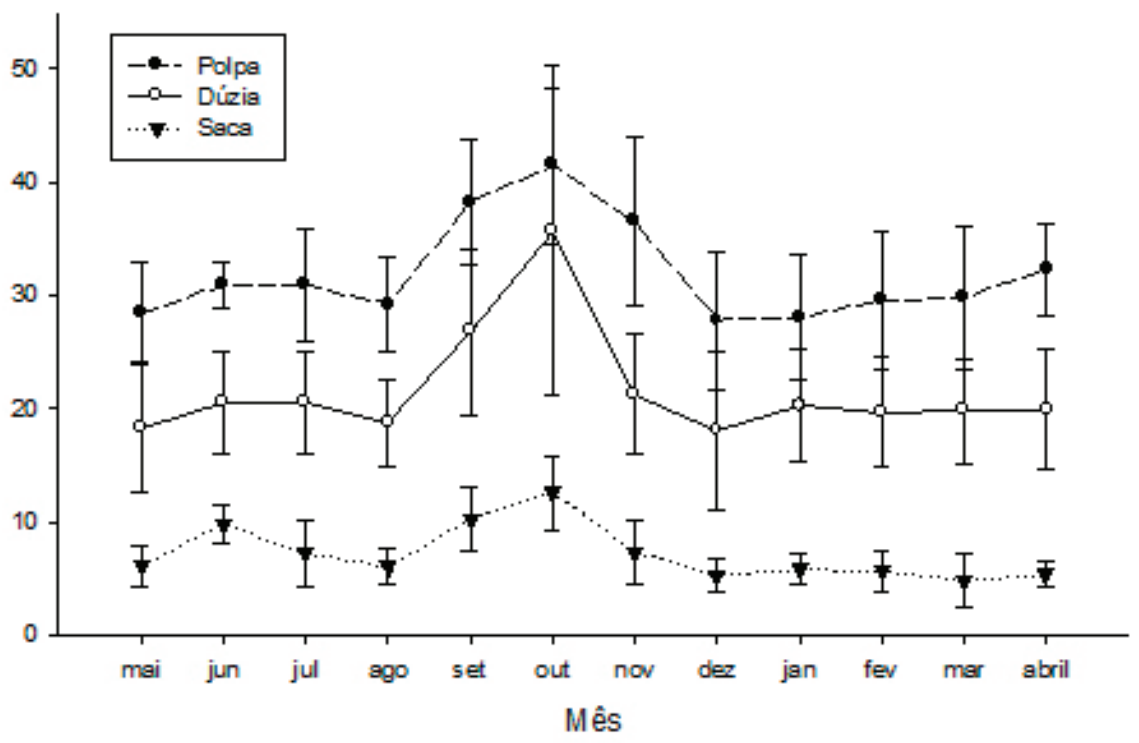

FIGURA 3 - Preço e valor agregado da polpa/kg na comercialização dos frutos de Astrocaryum aculeatum, em saca (41 $\pm 3 \mathrm{~kg})$, frutos selecionados para venda em dúzia $(0,7 \pm 0,2 \mathrm{~kg})$ ou pela venda da polpa, em feiras e mercados de Manaus-AM, entre maio/2011 e abril/2012.

TABELA 1 - Preço (R\$) mensal praticado e quantidade ( $\mathrm{t}$ ) mensal de Astrocaryum aculeatum comercializado entre maio de 2011 e abril de 2012.

\begin{tabular}{cccccc}
\hline & & unid. & Saca (unid.) & Fruto (dz.) & Polpa $(\mathrm{kg})$ \\
\hline \multirow{4}{*}{ Preço } & Média mensal & $\mathrm{R} \$$ & 77,91 & 3,79 & 31,47 \\
& mín - máx & $\mathrm{R} \$$ & $30,00-180,00$ & $2,50-5,00$ & $20,00-50,00$ \\
& Desv.Pad & $\mathrm{R} \$$ & 31,36 & 1,15 & 6,53 \\
& $\mathrm{CV}$ & $\%$ & $40 \%$ & $30 \%$ & $21 \%$ \\
\hline \multirow{4}{*}{ Quan- } & Média mensal & $\mathrm{t}$ & 30,7 & 14,3 & 16,4 \\
tidade & Mín - Máx & $\mathrm{t}$ & $13,6-42,8$ & $7,6-25,1$ & $6,0-22,4$ \\
& Desv. Pad. & $\mathrm{t}$ & 7,4 & 5,0 & 4,4 \\
& $\mathrm{CV}$ & $\%$ & $24 \%$ & $35 \%$ & $27 \%$ \\
& Total (12 meses) & $\mathrm{t}$ & 367,8 & 171,1 & 196,7 \\
\hline
\end{tabular}




\begin{tabular}{|c|c|c|c|c|c|c|c|c|c|c|c|c|c|c|c|c|c|c|c|c|c|c|}
\hline$\stackrel{\gtrless}{\Xi}$ & 号 & $\begin{array}{l}\stackrel{0}{2} \\
\stackrel{m}{-}\end{array}$ & $\begin{array}{l}\stackrel{0}{2} \\
\stackrel{\eta}{-}\end{array}$ & ภ̊ & ๙̊ & $\stackrel{R}{ }^{\circ}$ & $\stackrel{2}{2}$ & $\stackrel{2}{2}$ & ठें & $\stackrel{\circ}{+}$ & $\stackrel{\stackrel{\circ}{ }}{ }$ & 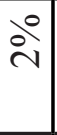 & $\stackrel{े}{\mathrm{~N}}$ & $\partial^{0}$ & $\partial^{0}$ & $0^{0}$ & $0^{0}$ & $0^{0}$ & $0^{0}$ & $\partial^{0}$ & $\partial^{\circ}$ & 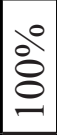 \\
\hline 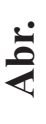 & $\stackrel{\infty}{0}^{\circ}$ & $\begin{array}{l}\stackrel{0}{ } \\
\text { ते }\end{array}$ & $\stackrel{2}{\stackrel{2}{二}}$ & & & & & $\begin{array}{l}\stackrel{0}{n} \\
\text { ते }\end{array}$ & $\stackrel{\circ}{\infty}$ & ळे & & & & & & & & & & & $\grave{\infty}_{\infty}^{0}$ & 吕 \\
\hline$\stackrel{\ddot{\Xi}}{\Sigma}$ & $\begin{array}{l}\stackrel{0}{ } \\
\text { ले }\end{array}$ & $\begin{array}{l}\stackrel{0}{\Delta} \\
\stackrel{\Delta}{2}\end{array}$ & $\stackrel{\circ}{\stackrel{\circ}{ }^{\prime}}$ & & ठ̊ํ. & $\hat{b}^{\circ}$ & $\hat{\delta}^{\circ}$ & & do & & & & & & & & & & & రి & & $\stackrel{\circ}{8}_{0}^{\circ}$ \\
\hline 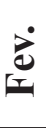 & $\begin{array}{l}\stackrel{0}{2} \\
\stackrel{2}{2}\end{array}$ & ১ें & $\stackrel{\circ}{\infty}$ & $\stackrel{\circ}{\infty}$ & $\stackrel{\circ}{\infty}$ & & $\begin{array}{l}\text { iे } \\
\text { ñ }\end{array}$ & $\stackrel{\circ}{\infty}$ & & & & $\begin{array}{l}i^{0} \\
\text { n }\end{array}$ & & $\stackrel{\circ}{\infty}$ & & & & & & & & $\stackrel{\circ}{8}$ \\
\hline$\underset{\Xi}{\stackrel{\Xi}{\Xi}}$ & ڤ̊ & $\stackrel{\circ}{\circ}$ & $8_{0}^{\circ}$ & $\hat{\theta}^{\circ}$ & ठి & ठి० & $\stackrel{\text { ̊̊ }}{\circ}$ & ठें & & bे & & & & & & ठें & & & & & & ᄋ̊ㅇㅇ \\
\hline$\stackrel{\dot{8}}{\circ}$ & $\stackrel{\circ}{\stackrel{\circ}{\sim}}$ & ڤे & i̊n & $\dot{8}^{\circ}$ & $\stackrel{\circ}{ \pm}$ & i̊ & $\stackrel{\circ}{\circ}$ & & & & ì & in & & & & & & in & & & & $\stackrel{\circ}{0}_{0}^{\circ}$ \\
\hline$\frac{\dot{z}}{z}$ & & $\stackrel{\circ}{\circ}$ & $\stackrel{8}{0}^{\circ}$ & $\begin{array}{l}\text { ठे } \\
\text { वे }\end{array}$ & & $\stackrel{\circ}{\circ}^{\circ}$ & $\stackrel{\circ}{\circ}$ & & $\stackrel{\circ}{\circ}$ & & & & & & 吕 & & & & & & & ᄋ̊ㅇㅇ \\
\hline$\dot{\Xi}$ & & & $\begin{array}{l}\stackrel{\circ}{ }^{\circ} \\
\end{array}$ & $\begin{array}{l}00 \\
\grave{\infty}\end{array}$ & & & & & & & & & & & & & & & & & & ᄋீ \\
\hline$\stackrel{\ddot{\oplus}}{\mathscr{\omega}}$ & & & $\begin{array}{l}\stackrel{0}{ } \\
\infty \\
n\end{array}$ & $\stackrel{\infty}{\infty}$ & $\stackrel{\circ}{\infty}$ & & & $\stackrel{\circ}{\infty}$ & $\begin{array}{l}\stackrel{0}{\infty} \\
\infty\end{array}$ & & & & & & & & $\stackrel{\circ}{\infty}$ & & & & & $\stackrel{\circ}{8}_{0}^{\circ}$ \\
\hline$\stackrel{8}{80}$ & & & $\begin{array}{l}\stackrel{0}{ } \\
\text { in } \\
\text { ते }\end{array}$ & & $\stackrel{\circ}{\stackrel{0}{=}}$ & $\begin{array}{l}\stackrel{0}{ } \\
\stackrel{2}{二}\end{array}$ & & $\begin{array}{l}\stackrel{0}{ } \\
\stackrel{m}{\eta}\end{array}$ & & $\begin{array}{l}\stackrel{0}{ } \\
\end{array}$ & bे & & $\begin{array}{l}\partial^{0} \\
\text { mे }\end{array}$ & & & & & & ठ̊ & & & ठ̊ㅇㅇ \\
\hline$\dot{\Xi}$ & $\stackrel{\text { 巳o }}{ \pm}$ & $\stackrel{\partial^{\circ}}{ \pm}$ & & & $\frac{\partial^{\circ}}{\sim}$ & $\stackrel{2}{2}$ & & $\stackrel{2}{\stackrel{\gamma}{ }^{\circ}}$ & $\frac{\partial^{\circ}}{{ }^{\circ}}$ & $\stackrel{2}{\stackrel{R}{ }^{\circ}}$ & $\stackrel{2}{R}^{0}$ & & & & & & & & & & & $\stackrel{\circ}{\circ}$ \\
\hline$\dot{\Xi}$ & ڤे & $\stackrel{\circ}{\stackrel{0}{\sigma}}$ & & & $8_{0}^{\circ}$ & $\begin{array}{l}\stackrel{0}{ } \\
\stackrel{m}{=}\end{array}$ & $\delta_{0}^{0}$ & $\begin{array}{l}\stackrel{0}{ } \\
\stackrel{m}{=}\end{array}$ & $\stackrel{\text { ڤ̊ }}{2}$ & ठ̊ํ & dे & & & & & & & & & & & $\stackrel{\circ}{8}$ \\
\hline$\stackrel{\ominus}{\stackrel{\ominus}{\pi}}$ & & $\begin{array}{l}\stackrel{0}{ } \\
\stackrel{2}{n}\end{array}$ & $\begin{array}{l}\stackrel{0}{ } \\
\end{array}$ & & $\begin{array}{l}\stackrel{0}{ } \\
\end{array}$ & $\begin{array}{l}\stackrel{0}{2} \\
\stackrel{2}{2}\end{array}$ & $\begin{array}{l}\stackrel{0}{ } \\
\text { ले }\end{array}$ & & & $\begin{array}{l}\stackrel{0}{m} \\
\stackrel{2}{n}\end{array}$ & & & $\begin{array}{l}\stackrel{0}{2} \\
\text { m }\end{array}$ & & & & & & & & & 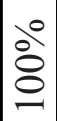 \\
\hline 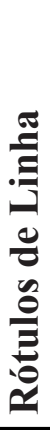 & 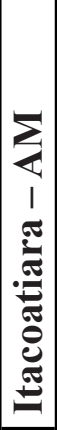 & 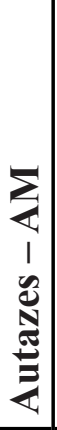 & 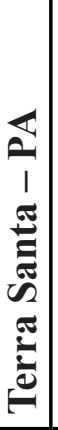 & 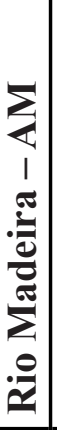 & 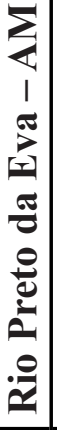 & 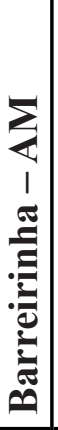 & 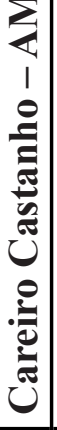 & 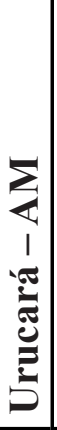 & 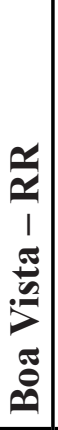 & 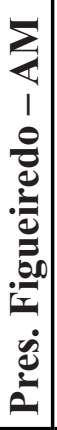 & 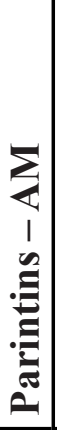 & 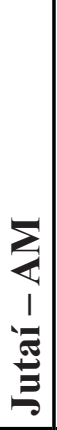 & 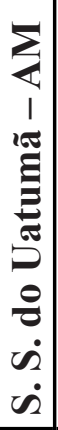 & 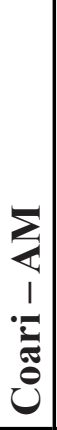 & 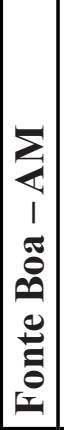 & 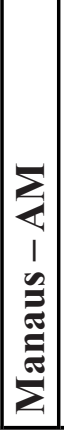 & 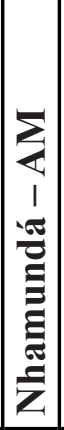 & 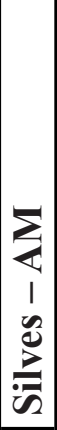 & 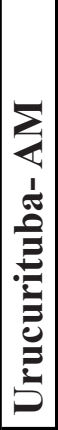 & 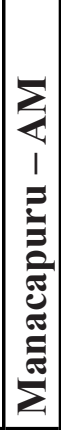 & 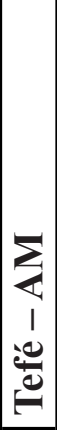 & \\
\hline
\end{tabular}


TABELA 3 - Preços históricos praticados em Manaus, deflacionados pelo Índice Geral de Preços (IGPDI), da polpa, com base em 2003, e da dúzia e saca, com base em 1995, de Astrocaryum aculeatum.

\begin{tabular}{lcccc}
\hline \multicolumn{1}{c}{ Trabalho } & Ano & Polpa (kg) & Fruto (dúzia) & Saca (unid.) \\
\hline Kahn e Moussa, 1999 & 1995 & - & $\mathrm{R} \$ 5,00$ & \\
Costa et al., 2005 & 2003 & $\mathrm{R} \$ 20,00$ & $\mathrm{R} \$ 4,42$ & $\mathrm{R} \$ 58,00$ \\
Schroth, 2004 & 2004 & $\mathrm{R} \$ 21,53$ & - & - \\
Rabelo, 2012 & 2010 & $\mathrm{R} \$ 58,81$ & $\mathrm{R} \$ 16,38$ & - \\
Presente trabalho & 2011 & $\mathrm{R} \$ 50,60$ & $\mathrm{R} \$ 15,78$ & $\mathrm{R} \$ 133,32$ \\
\hline
\end{tabular}

\section{AGRADECIMENTO}

Ao CNPq (Conselho Nacional de Desenvolvimento Científico e Tecnológico) e CAPES (Coordenação de Aperfeiçoamento de Pessoal de Nível Superior), pelo auxílio financeiro e às bolsas de mestrado e produtividade em pesquisa.

\section{REFERÊNCIAS}

ALMEIDA, A.N.; BITTENCOURT, A.M.; SANTOS A.J.; EISFELD, C.L.; SOUZA V.S. Evolução da produção e preço dos principais produtos florestais não madeireiros extrativos do Brasil. Cerne, Lavras, v. 15, n. 3, p. 282-287, 2009.

BELCHER, B.; SCHRECKENBERG, K. Commercialization of non-timber forest products: a reality check. Development Policy Review, London, v. 25, n 3, p. 355-377, 2007.

BRONDÍZIO, E.S.; SAFAR, C.A.M.; SIQUEIRA, A.D. The urban market of açaí fruit (Euterpe oleracea Mart.) and rural land use change: ethnographic insights into the role of price and land tenure constraining agricultural choices in the Amazon estuary. Urban Ecosystems, New York, v. 6, n1, p. 67-97, 2002.

CARDOSO, R. S.; FREITAS, C. E. C. Desembarque e esforço de pesca da frota pesqueira comercial de Manicoré (Médio Rio Madeira), Amazonas, Brasil. Acta Amazônica, Manaus, v. 37, n. 4, p. 605-612, 2007.
CLEMENT, C. R.; LLERAS, P. E.; VAN LEEUWEN, J. O potencial das palmeiras tropicais no Brasil: acertos e fracassos das últimas décadas. Revista Brasileira de Agrociências, Pelotas, v. 9, n. 1-2, p. 67-71, 2005.

COSTA, J.R. da; VAN LEEUWEN, J.; COSTA, J. A. Tucumã-do-Amazonas, Astrocaryum tucuma Martius. In: SHANLEY, P, O.; MADINA, G. (Ed.). Frutíferas e plantas úteis na vida amazônica. Belém: CIFOR, Imazon, 2005. 215-222.

FAO. Espécies forestales productoras de frutas y otros alimentos. 3. América Latina. Roma: SIDA/ FAO, 1987. 241p. (Estúdio FAO Montes, 44/3).

GENTIL, D.F.O.; FERREIRA, S.A.N. Morfologia da plântula em desenvolvimento de Astrocaryum aculeatum Meyer (Arecaceae). Acta Amazonica, Manaus, v. 35, n. 3, p. 339-344, 2005.

HENDERSON, A.; GALEANO, G.; BERNAL, R. Field guide to the palms of the Americas. New Jersey: Princepton University, 1995. p.200-207.

HOMMA, A. K. O. Uma tentativa de interpretação teórica do extrativismo amazônico. Acta Amazônica, Manaus, v.2, n.12, p. 251-255, 1982.

HOMMA, A. K. O.; CARVALHO, J. E. U.; MATOS, G. B.; MENEZES, A. J. E. A. Manejando a planta e o homem: os bacurizeiros do Nordeste Paraense e da Ilha de Marajó. Amazônia: Ciência \& Desenvolvimento, Belém. v. 2, n. 4, p. 119-135, 2007. 
IBGE - Instituto Brasileiro de Geografia e Estatística. Censo demográfico: Brasil, 2010. Rio de Janeiro, 2010.

KAHN, F. The genus Astrocaryum (Arecaceae). Revista Peruana de Biologia, Lima, v. 15, n. 1, p. 031- 048, 2008.

KHAN, F.; MOUSSA, F. Uso y potencial económico de dos palmas, Astrocaryum aculeatum Meyer y A. vulgare Martius, en la Amazonía brasileña. In: RIOS, M.; PEDERSEN, H. B. (Ed.). Uso y manejo de recursos vegetales. Quito: Abya-Yala, 1997. p. 101-116.

KAHN, F.; MOUSSA, F. Economic importance of Astrocaryum aculeatum (Palmae) in Central Brazilian Amazonia. Acta Botanica Venezuelica, Caracas, v. 22, n.1, p. 237-245, 1999.

LLERAS, L.; GIACOMETTI, D.C.; CORADIN, L. Areas criticas de distribucion de palmas en las Americas par colecta, evaluacion y conservacion. In: WORKSHOP ON UNDERUTILIZED PALMS OF TROPICAL AMERICA, 1983, Turrialba. Proceedings... San Jose: FAO, 1983. p.67-101.

LOPES, M.T.G.; MACÊDO, J.L.V.; LOPES, R.; VAN LEEUWEN, J.; RAMOS, S. L.F.; BERNARDES, L.G. Domesticação e melhoramento do Tucumã-do-Amazonas. In: BORÉM, A.; LOPES, M.T.G.; CLEMENT, C.R (Ed.). Domesticação e melhoramento. Viçosa: Universidade Federal de Viçosa, 2009. p. 424-441.
MARSHALL, E.; SCHRECKENBERG, K.; NEWTON, A.C. Commercialization of nontimber forest products: factors influencing success. Lessons learnt from Mexico and Bolivia and policy implications for decision-makers. International Forestry Review, Oxford, v.5, n. 2, p. 128-137, 2003.

NIMER, E. Climatologia da região norte: Introdução à climatologia dinâmica. Subsídios a geografia regional do Brasil. Revista Brasileira de Geografia, Rio de Janeiro, v. 34, n. 3, p. 124-153, 1972.

RABELO, A. Frutos nativos da Amazônia: comercializados nas feiras de Manaus-AM. Manaus: INPA, 2012. p. 361-372.

SCHROTH, G.; DA MOTA, M.S.S.; LOPES, R.;DE FREITAS, A.F. Extractive use, management and in situ domestication of a weedy palm, Astrocaryum aculeatum, in the central Amazon. Forest Ecology Management, Amsterdam, v. 202, p. 161-179, 2004. 